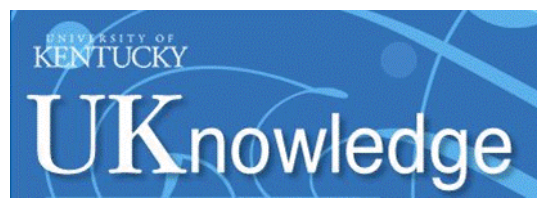

University of Kentucky

UKnowledge

10-10-1998

\title{
Temperature Fluctuations in Photoionized Nebulae. II. The Effect of Inhomogeneous Abundances
}

\author{
J. B. Kingdon \\ Space Telescope Science Institute \\ Gary J. Ferland \\ University of Kentucky, gary@uky.edu
}

Follow this and additional works at: https://uknowledge.uky.edu/physastron_facpub

Part of the Astrophysics and Astronomy Commons, and the Physics Commons

Right click to open a feedback form in a new tab to let us know how this document benefits you.

\section{Repository Citation}

Kingdon, J. B. and Ferland, Gary J., "Temperature Fluctuations in Photoionized Nebulae. II. The Effect of Inhomogeneous Abundances" (1998). Physics and Astronomy Faculty Publications. 117.

https://uknowledge.uky.edu/physastron_facpub/117

This Article is brought to you for free and open access by the Physics and Astronomy at UKnowledge. It has been accepted for inclusion in Physics and Astronomy Faculty Publications by an authorized administrator of UKnowledge. For more information, please contact UKnowledge@lsv.uky.edu. 


\section{Temperature Fluctuations in Photoionized Nebulae. II. The Effect of Inhomogeneous Abundances}

\section{Digital Object Identifier (DOI)}

http://dx.doi.org/10.1086/306236

\section{Notes/Citation Information}

Published in The Astrophysical Journal, v. 506, no. 1, p. 323-328.

(c) 1998. The American Astronomical Society. All rights reserved. Printed in the U.S.A.

The copyright holder has granted permission for posting the article here. 
The AstrophysicAL Journal, 506:323-328, 1998 October 10

(C) 1998. The American Astronomical Society. All rights reserved. Printed in U.S.A.

\title{
TEMPERATURE FLUCTUATIONS IN PHOTOIONIZED NEBULAE. II. THE EFFECT OF INHOMOGENEOUS ABUNDANCES
}

\author{
J. B. KINGDON \\ Space Telescope Science Institute, 3700 San Martin Drive, Baltimore, MD 21218 \\ AND \\ G. J. FERLAND \\ Department of Physics and Astronomy, University of Kentucky, Lexington, KY 40506; kingdon@stsci.edu; gary@.pop.uky.edu \\ Received 1998 February 3; accepted 1998 May 19
}

\begin{abstract}
Recent abundance determinations based on recombination lines in several emission-line nebulae yield ionic abundances several times larger than those derived from forbidden lines. These results cast uncertainty over all abundance determinations in such objects. One possible explanation for these discrepancies frequently cited in the literature is the presence of chemical inhomogeneities. We have run a series of photoionization models to examine what effect such inhomogeneities will have on the resulting temperature structure of nebulae. We then derive abundances from these models, utilizing Peimbert's $t^{2}$ formalism. Our results suggest that, although chemical inhomogeneities may produce nonnegligible biases in abundance determinations in a small number of objects, it is highly unlikely that they can resolve the observed discrepancy for most nebulae. We also stress the importance of continued high spatial resolution observations in nebulae to clarify the presence or absence of inhomogeneities in gaseous nebulae.
\end{abstract}

Subject headings: ISM: abundances - planetary nebulae: general

\section{INTRODUCTION}

Accurate abundances are essential for a host of astrophysical problems, including stellar and galactic chemical evolution (Shields 1990; Aller 1990) and the primordial helium abundance (Olive, Steigman, \& Skillman 1997). In light of this, several recent abundance determinations in a number of $\mathrm{H}$ II regions and planetary nebulae (PNs) have caused much concern. Using recombination lines, Peimbert, Storey, \& Torres-Peimbert (1993) obtained O abundances in two $\mathrm{H}$ il regions and a PN that were roughly $50 \%$ greater than the value obtained by the more traditional forbiddenline method. Similarly, Liu et al. (1995) determined abundances of $\mathrm{C}, \mathrm{N}$, and $\mathrm{O}$ in the PN NGC 7009 using recombination lines that were factors of 4-6 larger than the corresponding forbidden-line abundances. Barlow et al. (in preparation) obtained discrepancies of approximately the same order of magnitude for six other PNs. Clearly, these results need to be understood before any nebular abundances can be viewed with certainty.

These results have renewed interest in the concept of temperature fluctuations, originally formulated by Peimbert (1967). In a nonisothermal nebula, the emissivity of a collisionally excited line will be preferentially weighted toward regions of higher temperature. This will lead to an underestimate of abundances determined from these lines if temperature fluctuations are not accounted for. Conversely, abundances based on recombination lines would be overestimated; however, because of the relatively weak temperature dependence of these lines, this effect is significantly less than that for the forbidden lines.

While the concept of temperature fluctuations is straightforward, finding a physical mechanism capable of producing sufficiently large fluctuations has proved elusive. Kingdon \& Ferland (1995, hereafter Paper I) and
Gruenwald \& Viegas (1995) have shown that standard photoionization models cannot produce values of Peimbert's $t^{2}$ parameter large enough to explain the observed discrepancies. Maciejewski, Mathis, \& Edgar (1996) investigated the importance of the transition layer between the visible nebula and the hot plasma that confines it. [O III] $\lambda 4363$ produced in this layer would decrease the derived $(\mathrm{O} / \mathrm{H})$ abundance. They conclude that the amount of [O III] $\lambda 4363$ produced in this zone is too small to account for the observed discrepancy in the $\mathrm{O}$ abundance. Some progress has been made by Pérez (1997), who produced relatively large $t^{2}$ in some starburst models.

One possible solution that has been frequently proposed in the literature (e.g., Peimbert 1995) is the presence of chemical inhomogeneities within the nebula. These inhomogeneities would affect the temperature structure both directly, by altering the amount of coolants, and indirectly via opacity effects. Torres-Peimbert, Peimbert, \& Peña (1990) invoked variable abundances in order to reconcile a discrepancy between the value of $\left(\mathrm{C}^{+2} / \mathrm{H}^{+}\right)$derived from $\mathrm{C}$ III] $\lambda 1909$ and that obtained from C II $\lambda 4267$ in the PN NGC 4361. They found that a two-zone model having a C-rich inner zone with the carbon abundance roughly 80 times larger than that in the outer zone could account for the observed intensity of both lines.

Unfortunately, the evidence for abundances varying with position in real nebulae remains somewhat uncertain. Abundance differences between the main nebula and the outer halo were noted by Manchado \& Pottasch (1989) in the PNs NGC 6543 and NGC 6826. The N/O ratio was found to increase outward in the PNs NGC 6751 (Chu et al. 1991) and M1-75 (Guerrero, Stanghellini, \& Manchado 1995), although the data did not permit evaluation of changes in either $\mathrm{N}$ or $\mathrm{O}$ individually. Variations were also 
observed in A78 (Manchado, Pottasch, \& Mampaso 1988) and A30 and A58 (Guerrero \& Manchado 1996), three of the so-called "born again" planetaries. Conversely, Corradi et al. (1997) found no evidence of variation for $\mathrm{He}, \mathrm{O}, \mathrm{N}, \mathrm{Ne}$, and $\mathrm{Ar}$ in the bipolar PN IC 4406, and although recent spatially resolved observations of NGC 4361 by Liu (1998) confirm the discrepancy in the $\mathrm{C}$ abundance noted by Torres-Peimbert et al. (1990), the C abundance measured from recombination lines is found to be constant across the nebula.

Detection of abundance inhomogeneities is particularly difficult, as it requires not only high spatial resolution but also multiwavelength spectroscopy to ensure accuracy. This is due to the fact that many elements are only represented by a few ionization stages in the optical part of the spectrum, requiring the unobserved stages to be estimated via ionization correction factors (ICFs). These ICFs can be a major source of uncertainty (see Alexander \& Balick 1997 for a detailed discussion). Indeed, although Corradi et al. (1997) did measure an increase in $\mathrm{S} / \mathrm{H}$ with radius in IC 4406, they argue that this result may in fact be an artifact of the uncertainties in the ICF for sulfur.

On the theoretical side, the picture is even less clear. Although we have a reasonable understanding of the changes in surface abundances in asymptotic giant branch (AGB) stars brought about by the various dredge-up events (see Vassiliadis \& Wood 1993), uncertainties in the mechanism that produces PNs makes it difficult to translate this information into abundances in the ejected material. The current prevailing view of PN formation posits that the ejecta is fed by mass loss through a stellar wind occurring during the thermal pulsing AGB phase. If mass loss during different thermal pulse cycles leads to discrete shells of gas, then one would expect abundance differences to exist between the ejected shells because of the varying efficiency of the third dredge-up process from one cycle to the next. Additionally, if the timescale for convective mixing is much longer than the timescale for mass loss during a thermal pulse, then it would be possible to have varying abundances within each individual shell. There also exists a class of objects referred to as "born again" PNs (Iben et al. 1983), in which a final thermal pulse occurs during the post-AGB phase causing the star to undergo the AGB phase again. In this case, the gas ejected during the second AGB phase should have abundances very different from those ejected during the first.

In this paper, we investigate the effect of inhomogeneities on temperature structure and derived abundances by use of a series of photoionization models. In $\S 2$ we describe the models and present our calculations. We discuss the results and the implications for resolving the abundance discrepancy in $\S 3$. For convenience, we have included a brief description of the $t^{2}$ formalism and all appropriate formulae in the Appendix.

\section{CALCULATIONS}

In order to examine the effect of abundance inhomogeneities, we utilize the photoionization code CLOUDY (Ferland 1996), which consistently maintains both ionization and thermal balance. We consider four baseline models using two values of the hydrogen density $\left(\log n_{\mathrm{H}}=3,5\right)$ and two stellar temperatures $\left(\log T_{\text {eff }}=4.5,5.3\right)$. For all models, the ionizing source is a blackbody with a total luminosity of $10^{38} \mathrm{ergs} \mathrm{s}^{-1}$. The total hydrogen density is constant with radius and the filling factor is set to 1 . The inner face of the gas is located $10^{17} \mathrm{~cm}$ from the blackbody source. The results are not dependent on this value. All models are terminated at the $\mathrm{H}$ ionization front. The most recent version of the code allows the user to vary any of the abundances with radius by inputting radius/abundance pairs. For simplicity, we consider only the effects of varying the oxygen abundance, as this element is the primary coolant in most nebulae. The abundances of all other elements are taken from the stored PN abundance set in CLOUDY, which represents a weighted average of PN abundances given in Aller \& Czyzak (1983) and Khromov (1989). Grains based on unpublished work by K. Volk on post-AGB stars are included.

Since, as will be discussed later, we will need to consider the greatest extremes of conditions, some of the nebulae considered below have very high oxygen abundances. In light of this, a few words on the accuracy of CLOUDY in this regime is warranted. CLOUDY has always been designed to simulate metal-rich environments. The opacities of all elements are explicitly included. Their effects on the heating (by photoionization) and cooling (mainly by collisionally excited lines) are treated as well. Ferland et al. (1998) show cases where a cloud totally dominated by heavy elements is exposed to various blackbodies and goes to the appropriate thermodynamic limits. The accuracy of such metal-dominated simulations is hard to judge. The photoionization cross sections are fairly well known for secondrow elements and more highly charged species. The lack of accurate dielectronic recombination rate coefficients for third-row elements is probably the greatest uncertainty in the overall equilibrium. Ferland et al. (1998) discuss this and other uncertainties.

\subsection{Gradual Models}

We first consider models in which the oxygen abundance varies gradually over the entire ionized zone. As discussed in $\S 1$, such a situation could occur in PNs if the timescale for convective mixing is much longer than that for mass loss. We begin by studying the effects of a relatively small abundance variation; specifically, we consider a factor of 5 change in the $\mathrm{O}$ abundance across the nebula. For each of the four baseline models, we calculate results for both an increase and a decrease with radius by this amount. For the models in this and all subsequent sections, we take the CLOUDY stored $\mathrm{PN}$ abundance set $\mathrm{O} / \mathrm{H}=4.4 \times 10^{-4}$ as a mean value. Thus, the four increasing models computed in this section will have $\mathrm{O} / \mathrm{H}=1.97 \times 10^{-4}$ at the ionized face, increasing to $\mathrm{O} / \mathrm{H}=9.84 \times 10^{-4}$ at the $\mathrm{H}$ ionization front. These numbers are reversed for the four decreasing models. The variation with radius in each model is set so that the $\log$ of $\mathrm{O} / \mathrm{H}$ varies linearly with the $\log$ of the depth into the cloud.

The results of the calculations described above are presented in Figure 1 and Table 1. The quantity "depth" in this figure is defined as the distance from the ionized face of the cloud to a point within the cloud. For each of the four baseline models, Figure 1 displays the temperature structure for $\mathrm{O} / \mathrm{H}$ increasing by a factor of 5 (solid line), $\mathrm{O} / \mathrm{H}$ decreasing by a factor of 5 (dashed line), and a constant $\mathrm{O} / \mathrm{H}=4.4 \times 10^{-4}$ (dotted line). These models behave qualitatively as expected; namely, a higher (lower) oxygen abundance at a given depth results in a lower (higher) temperature there. Table 1 lists both $t^{2}\left(\mathrm{H}^{+}\right)$and $t^{2}\left(\mathrm{O}^{+2}\right)$ for 

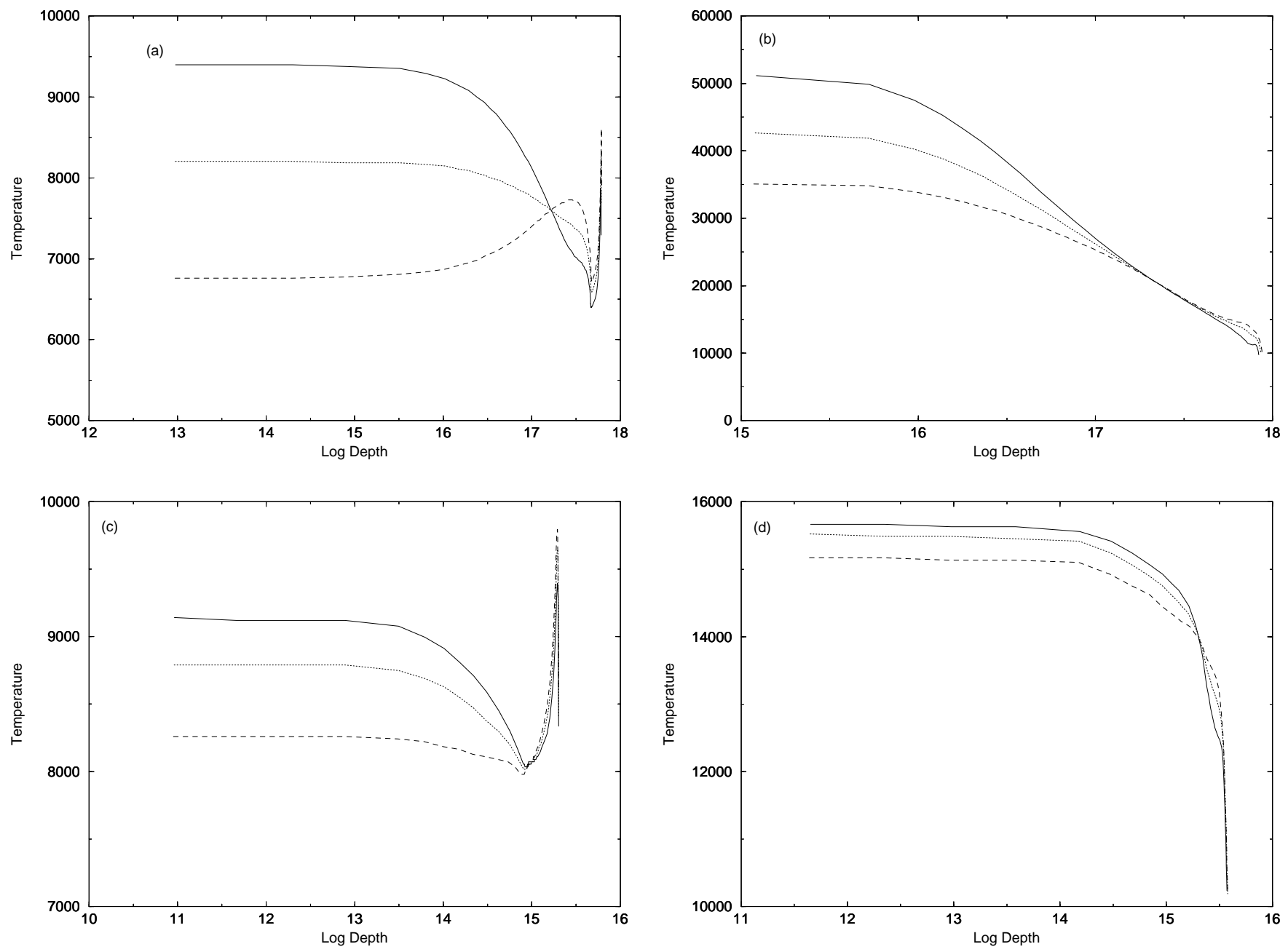

Fig. 1.-Temperature structure for gradual models. The solid line is for $\mathrm{O} / \mathrm{H}$ increasing by a factor of 5 , the dashed line for $\mathrm{O} / \mathrm{H}$ decreasing by a factor of 5, and the dotted line for constant $\mathrm{O} / \mathrm{H}=4.4 \times 10^{-4}$. (a) Model with $\log n_{\mathrm{H}}=3, \log T_{\text {eff }}=4.5$. (b) Model with $\log n_{\mathrm{H}}=3, \log T_{\text {eff }}=5.3$. (c) Model with $\log$ $n_{\mathrm{H}}=5, \log T_{\text {eff }}=4.5,(d)$ Model with $\log n_{\mathrm{H}}=5, \log T_{\text {eff }}=5.3$.

all of these cases. We note that for the models in this and all subsequent sections, the effect of the abundance variations on the ionization structure is minor.

\subsection{Abrupt Models}

We next consider the case in which the oxygen abundance varies abruptly over a relatively small radius. These models can be thought of as consisting of two shells, with the oxygen abundance constant within each shell, but differing between the two. For stability of the models, it was necessary to include a transition region in which the oxygen abundance varies smoothly from its value in the inner shell to that in the outer shell. The radius of this region is typically $2 \%-3 \%$ of the total cloud thickness. For all models considered here, we have arbitrarily chosen the transition region to occur at half the Strömgren depth. Thus, both shells have the same thickness. For illustrative purposes, we again consider a factor of 5 difference in $\mathrm{O} / \mathrm{H}$ between the shells, and again use $\mathrm{O} / \mathrm{H}=4.4 \times 10^{-4}$ as a mean value. Therefore, all four decreasing models have $\mathrm{O} / \mathrm{H}=9.84 \times 10^{-4}$ in the inner shell and $\mathrm{O} / \mathrm{H}=1.97 \times$ $10^{-4}$ in the outer shell, with these values reversed for the increasing models.
We present our results in Table 1 and Figure 2. The line styles for Figure 2 are identical to those of Figure 1. In general, the $t^{2}$ values are larger for these models than for the analogous gradual models.

\subsection{Maximizing $t^{2}$}

It is clear from Table 1 not only that the models of the last two sections do not produce large $t^{2}$ values but that they also do not differ significantly from the analogous constant-abundance models. Although it is conceivable that some combination of model parameters could produce $t^{2}$ values much larger than those obtained here, our results suggest that small abundance variations cannot in general produce large temperature fluctuations.

This then prompts the question, how large a variation in the oxygen abundance is necessary to produce large $t^{2}$ ? Since our eventual goal is to create large discrepancies in the derived $\mathrm{O} / \mathrm{H}$ abundance ratio, and as this is essentially equivalent to producing large $t^{2}\left(\mathrm{O}^{+2}\right)$, we determine in this section the minimum abundance variation needed to obtain a value of $t^{2}\left(\mathrm{O}^{+2}\right)=0.05$ for each of our four baseline models. We note here that for temperature fluctuations of this magnitude, some error is introduced by stopping the Taylor series expansion (see Appendix) at second order (see 
TABLE 1

Results for Small Abundance Variations

\begin{tabular}{|c|c|c|c|c|}
\hline $\log n_{\mathrm{H}}$ & $\log T_{\text {eff }}$ & Increasing/Decreasing & $t^{2}\left(\mathrm{H}^{+}\right)$ & $t^{2}\left(\mathrm{O}^{+2}\right)$ \\
\hline \multicolumn{5}{|c|}{ Constant Models } \\
\hline $3 \ldots \ldots$ & 4.5 & $\cdots$ & $3.05(-3)$ & $8.29(-4)$ \\
\hline $3 \ldots \ldots$ & 5.3 & $\ldots$ & $3.66(-2)$ & $4.03(-3)$ \\
\hline $5 \ldots \ldots$ & 4.5 & $\ldots$ & $2.30(-3)$ & $6.37(-4)$ \\
\hline $5 \ldots \ldots$ & 5.3 & $\ldots$ & $4.73(-3)$ & $2.65(-3)$ \\
\hline \multicolumn{5}{|c|}{ Gradual Models } \\
\hline $3 \ldots \ldots$ & 4.5 & Increasing & $3.94(-3)$ & $3.47(-3)$ \\
\hline $3 \ldots \ldots$ & 4.5 & Decreasing & $3.28(-3)$ & $7.70(-4)$ \\
\hline $3 \ldots \ldots$ & 5.3 & Increasing & $5.77(-2)$ & $5.84(-3)$ \\
\hline $3 \ldots \ldots$ & 5.3 & Decreasing & $2.70(-2)$ & $3.57(-3)$ \\
\hline $5 \ldots \ldots$ & 4.5 & Increasing & $1.78(-3)$ & $1.24(-3)$ \\
\hline $5 \ldots \ldots$ & 4.5 & Decreasing & $3.24(-3)$ & $6.92(-5)$ \\
\hline $5 \ldots \ldots$ & 5.3 & Increasing & $6.73(-3)$ & $4.32(-3)$ \\
\hline $5 \ldots \ldots$ & 5.3 & Decreasing & $3.24(-3)$ & $1.37(-3)$ \\
\hline \multicolumn{5}{|c|}{ Abrupt Models } \\
\hline $3 \ldots \ldots$ & 4.5 & Increasing & $1.17(-2)$ & $1.59(-2)$ \\
\hline $3 \ldots \ldots$ & 4.5 & Decreasing & $5.60(-3)$ & $2.63(-3)$ \\
\hline $3 \ldots \ldots$ & 5.3 & Increasing & $6.86(-2)$ & $4.79(-3)$ \\
\hline $3 \ldots \ldots$ & 5.3 & Decreasing & $2.22(-2)$ & $4.13(-3)$ \\
\hline $5 \ldots \ldots$ & 4.5 & Increasing & $1.82(-3)$ & $1.01(-3)$ \\
\hline $5 \ldots \ldots$ & 4.5 & Decreasing & $4.39(-3)$ & $2.54(-4)$ \\
\hline $5 \ldots \ldots$ & 5.3 & Increasing & $1.07(-2)$ & $5.33(-3)$ \\
\hline $5 \ldots \ldots$ & 5.3 & Decreasing & $3.37(-3)$ & $2.31(-3)$ \\
\hline
\end{tabular}

NoTE.-The notation $a(b)$ here means $a \times 10^{b}$.
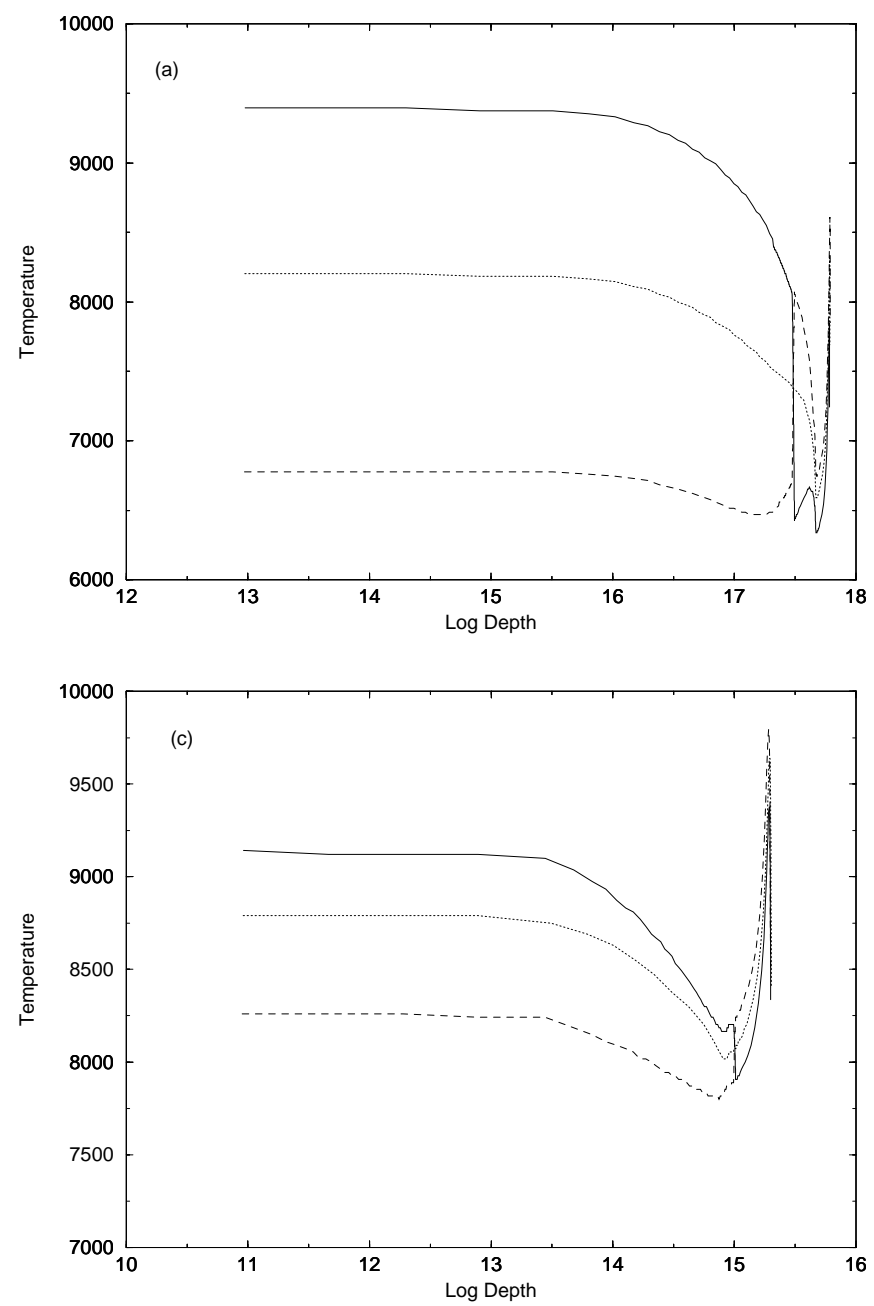

Rubin et al. 1998). The neglect of these higher order terms does not affect the conclusions reached here. Based on the results of Table 1 and the fact that maximizing $t^{2}\left(\mathrm{O}^{+2}\right)$ requires a significant temperature change over the $\mathrm{O}^{+2}$ zone, we use the abrupt models rather than the gradual ones. Since the location of the transition region in these models will affect the derived $t^{2}\left(\mathrm{O}^{+2}\right)$, we allowed this to vary in order to produce the maximum possible value for a given abundance change. We consider both increasing and decreasing models, again requiring $\mathrm{O} / \mathrm{H}=4.4 \times 10^{-4}$ to be the geometric mean.

Using the above prescription, we found that it was impossible to achieve $t^{2}\left(\mathrm{O}^{+2}\right)=0.05$ for the two highdensity models, even with a factor of $10^{6}$ difference in the oxygen abundance between the inner and outer shells. This is because the high density causes the gas to go neutral quickly, resulting in a very thin Strömgren sphere, as evidenced by the depth scale for the high-density models in Figures 1 and 2. This in turn reduces $t^{2}\left(\mathrm{O}^{+2}\right)$ through the volume element (see eq. [A3]). Essentially, the physical volume occupied by $\mathrm{O}^{+2}$ in these models is too small for any substantial temperature change to occur.

We were successful in producing $t^{2}\left(\mathrm{O}^{+2}\right)=0.05$ for the $\log n_{\mathrm{H}}=3, \log T_{\text {eff }}=5.3$ baseline model by having an oxygen abundance in the outer shell a factor of $\sim 5000$ larger than that in the inner shell. For the $\log n_{\mathrm{H}}=3, \log$ $T_{\text {eff }}=4.5$ model, a decrease in the oxygen abundance in the
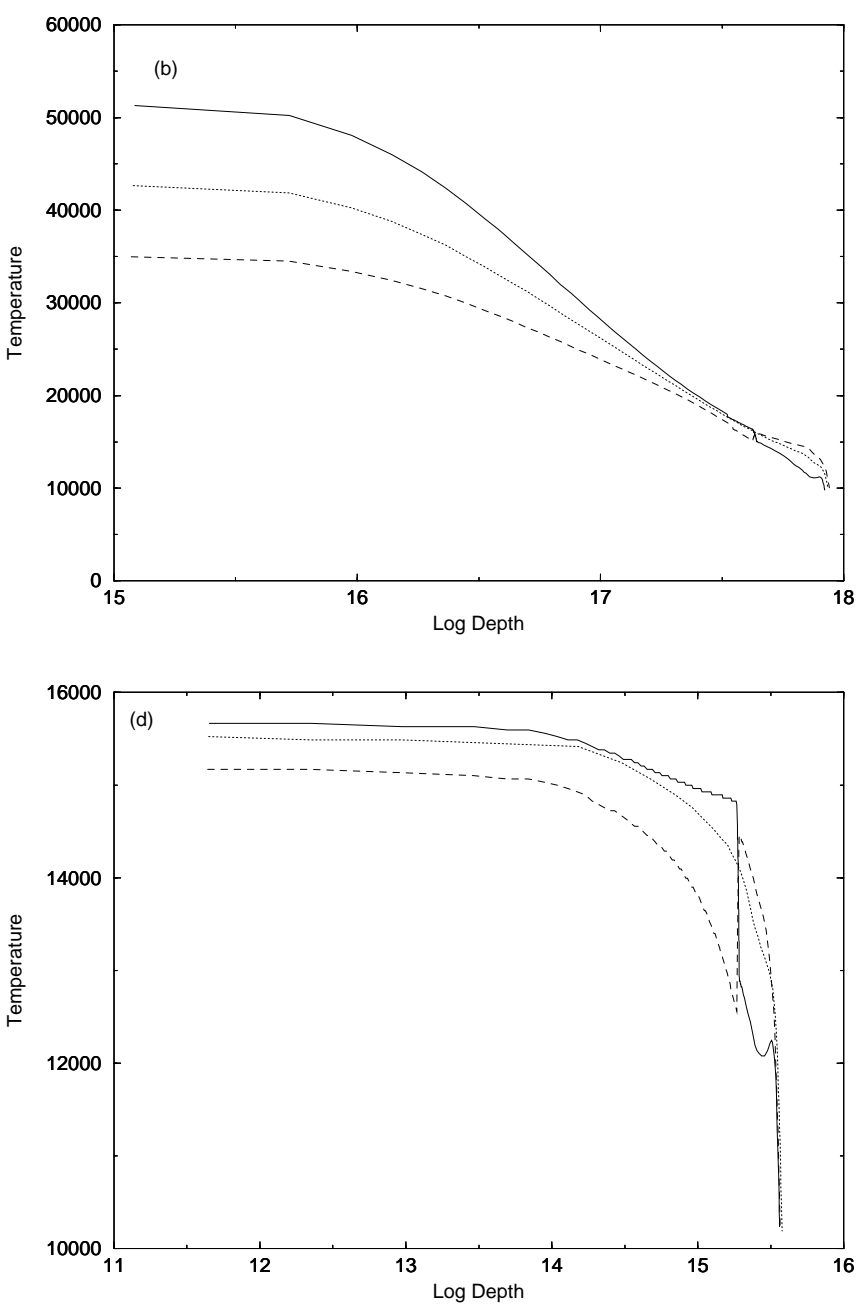

Fig. 2.-Same as Fig. 1, but for abrupt models 
outer shell by a factor of $\sim 20$ was required. At first glance, these results might seem counterintuitive, as Figures 1 and 2 show that the constant $(3,5.3)$ model has a very steep temperature falloff, while the constant $(3,4.5)$ model has a relatively flat temperature structure. The explanation again lies in the volume occupied by $\mathrm{O}^{+2}$. For the $(3,5.3)$ model, the high stellar temperature causes $\mathrm{O}$ to be present mainly as $\mathrm{O}^{+3}$ and $\mathrm{O}^{+4}$, with the $\mathrm{O}^{+2}$ zone relatively small. In the (3, 4.5) model, on the other hand, the $\mathrm{O}^{+2}$ zone is much larger, nearly coincident with the entire $\mathrm{H}^{+}$zone. It is therefore easier to produce large $t^{2}\left(\mathrm{O}^{+2}\right)$ in the latter model. Simply stated, although large $t^{2}\left(\mathrm{O}^{+2}\right)$ generally implies large $t^{2}\left(\mathrm{H}^{+}\right)$, the converse is not necessarily true.

We now wish to determine the actual oxygen abundance that would be inferred from these two models. Our method is identical to that in Paper I. We calculate the ionic abundance ratio $\mathrm{O}^{+2} / \mathrm{H}^{+}$in two ways: first, using equation (A5), which explicitly takes the effect of temperature fluctuations into account, and second, using the "observed" $T(\mathrm{O}$ III) (derived from model-predicted line intensities) in place of both $T_{\mathrm{H} \beta}$ and $T_{5007}$ in the same equation. The latter method is standard practice for deriving abundances, and implicitly assumes that no temperature fluctuations are present.

We list our results in Table 2, which gives all $t^{2}$ values and temperatures from the models necessary to compute the abundances. The $\mathrm{O}^{+2} / \mathrm{H}^{+}$value calculated by ignoring temperature fluctuations is given in column (9), the value taking temperature fluctuations into account is given in column (10), and the ratio of these two is given is column (11). For both models, ignoring the temperature fluctuations results in an underestimate of $\mathrm{O}^{+2} / \mathrm{H}^{+}$by roughly a factor of 2 .

\section{DISCUSSION}

Before examining the results of the previous section, we wish first to reconsider the primary assumption in all of our models; namely, that only the oxygen abundance varies while all the other elements remain constant. We used the $(3,4.5)$ model with the gradual scenario and a small abundance variation, and examined the effect of varying other abundant metal in lockstep with oxygen. Our results confirm that for this model, oxygen is by far the most important for determining the temperature structure; only $\mathrm{N}$ and $\mathrm{S}$ also made nonnegligible contributions. The calculated $t^{2}\left(\mathrm{O}^{+2}\right)$ with all metals varying was only a factor of $\sim 2$ larger than the model with only oxygen varying. Although the magnitude of this effect will undoubtedly increase with the size of the variation, there is no a priori reason to believe that other metals will vary in sync with oxygen. In fact, a model with, say, nitrogen decreasing and oxygen increasing with radius could actually reduce the derived $t^{2}\left(\mathrm{O}^{+2}\right)$ obtained from a model with oxygen varying alone. Thus, it is doubtful that the addition of more elements would substantially affect our conclusions here.

The results of this paper strongly suggest that abundance inhomogeneities cannot produce temperature fluctuations large enough to resolve the observed discrepancies. We found that it was impossible to achieve large $t^{2}$ for our high-density models with any reasonable variation. The factor of $\sim 5000$ change in the oxygen abundance for the $(3$, 5.3) model also seems unattainable based on AGB dredgeup models (Vassiliadis \& Wood 1993). Only the factor of $\sim 20$ change for the $(3,4.5)$ model seems even mildly plaus$\mathrm{ible}$, and this value is probably also too large. One must also keep in mind that these models were optimized to produce the largest possible $t^{2}\left(\mathrm{O}^{+2}\right)$ for a given abundance variation, within the constraints of our assumptions. Thus, the factor of $\sim 20$ is a lower limit for these parameters.

For completeness, we note that in deriving $T(\mathrm{O}$ III) from model-predicted intensities, we used the collisional component of [O III] $\lambda 4363$. For most of the models presented here, this is identical to the total [O III] $\lambda 4363$. However, for the $(3,5.3)$ model with a factor of $\sim 5000$ change, the large oxygen abundances in the inner region of the nebula produce very low temperatures. This in turn produces a nonnegligible recombination component to the line. If we had calculated $T(\mathrm{O}$ III) from the total [O III] $\lambda 4363$ intensity (collisional plus recombination), we would obtain a value $50 \%$ larger than that given in Table 2 . This would decrease the derived value of $\mathrm{O}^{+2} / \mathrm{H}^{+}$in column (9). Conversely, this implies that one could produce a factor of 2 discrepancy with a smaller abundance variation for this model. However, the required variation would still be well over 2 orders of magnitude, and therefore still physically unrealistic.

While abundance inhomogeneities do not appear capable of resolving the abundance discrepancy, our results suggest that, provided they exist, they can cause nonnegligible effects for some objects with low density and stellar temperature. Unfortunately, it is difficult to make any concrete predictions because of the aforementioned lack of definite observational evidence for such inhomogeneities. We again stress the importance of high-resolution, multiwavelength observations to clarify this issue.

The authors are grateful to E. Vassiliadis for valuable discussions and comments. This work was partially supported by NASA through grant number GO-05638.01-96A from the Space Telescope Science Institute, which is operated by the Association of Universities for Research in Astronomy, Inc. under NASA contract NAS 5-26555. Research in nebular astrophysics at the University of Kentucky is supported by the National Science Foundation through award AST 96-17083.

TABLE 2

Abundance Results

\begin{tabular}{ccccccccccc}
\hline \hline Model & $T(\mathrm{O}$ III) & $T_{0}\left(\mathrm{O}^{+2}\right)$ & $t^{2}\left(\mathrm{O}^{+2}\right)$ & $T_{5007}$ & $T_{0}\left(\mathrm{H}^{+}\right)$ & $t^{2}\left(\mathrm{H}^{+}\right)$ & $T_{\mathrm{H} \beta}$ & $\mathrm{O}^{+2} / \mathrm{H}^{+\mathrm{a}}$ & $\mathrm{O}^{+2} / \mathrm{H}^{+\mathrm{b}}$ & $R$ \\
\hline $1^{\mathrm{c}} \ldots \ldots$ & $8.186(3)$ & $6.548(3)$ & $5.63(-2)$ & $6.863(3)$ & $7.752(3)$ & $1.01(-2)$ & $7.679(3)$ & $2.690(-5)$ & $5.255(-5)$ & 1.95 \\
$2^{\mathrm{d}} \ldots \ldots$ & $6.713(3)$ & $4.609(3)$ & $4.85(-2)$ & $4.968(3)$ & $1.516(4)$ & $1.52(-1)$ & $1.301(4)$ & $1.214(-3)$ & $2.778(-3)$ & 2.29 \\
\hline
\end{tabular}

NoTE.-The notation $a(b)$ here means $a \times 10^{b}$.

${ }^{a}$ Derived from $T(\mathrm{O}$ III).

${ }^{b}$ Derived from $T_{5007}$ and $T_{\mathrm{H} \beta}$.

${ }^{c}$ Model 1 has $\log n_{\mathrm{H}}=3, \log T_{\text {eff }}=4.5$, and a factor of 20 decrease in $\mathrm{O} / \mathrm{H}$ with radius.

${ }^{\mathrm{d}}$ Model 2 has $\log n_{\mathrm{H}}=3, \log T_{\text {eff }}=5.3$, and a factor of 5000 increase in $\mathrm{O} / \mathrm{H}$ with radius. 


\section{APPENDIX A \\ FORMALISM FOR TEMPERATURE FLUCTUATIONS}

In this Appendix, we give a brief overview of the $t^{2}$ formalism, including pertinent equations for the derivation of abundances. A much more detailed treatment is provided by Peimbert (1967), which includes the derivation of most of the formulae presented here.

The intensity of a recombination line or a forbidden line below the critical density limit can be written

$$
I(\lambda)=\int n_{e} n_{\mathrm{in}} \epsilon(T) d V,
$$

where $n_{e}$ and $n_{\text {ion }}$ represent the electron and ion densities, respectively, and $\epsilon(T)$ is a function of temperature. We may then expand the $\epsilon(T)$ term in a Taylor series about a mean temperature $T_{0}$. If the variation of the temperature over the volume considered in the integral above is relatively small, then we can truncate this series so that we retain only terms up to second order. Let us then define a mean temperature $T_{0}$ by

$$
T_{0}=\frac{\int T n_{e} n_{\text {ion }} d V}{\int n_{e} n_{\text {ion }} d V}
$$

We further define an additional parameter

$$
t^{2}=\frac{\int\left(T-T_{0}\right)^{2} n_{e} n_{\text {ion }} d V}{T_{0}^{2} \int n_{e} n_{\text {ion }} d V} .
$$

Using this formalism, any temperature or function of temperature can be written in terms of the two parameters $T_{0}$ and $t^{2}$, subject to the assumptions described above. For example, the commonly used temperature derived from the [O III] $\lambda 5007 /$ $\lambda 4363$ ratio can be written

$$
T(\mathrm{O} \text { III })=T_{0}\left(\mathrm{O}^{+2}\right)\left\{1+\left[\frac{91380}{T_{0}\left(\mathrm{O}^{+2}\right)}-3\right] \frac{t^{2}\left(\mathrm{O}^{+2}\right)}{2}\right\} .
$$

We note that because of a typographical error, this equation was printed incorrectly in Paper I (eq. [6] of that paper). The difference is negligible, and since all values of $T(\mathrm{O}$ III) in that paper were derived from the model-predicted emission-line intensities, the results of Paper I are unchanged.

The ionic abundance ratio $\left(\mathrm{O}^{+2} / \mathrm{H}^{+}\right)$based on the intensity ratio of $[\mathrm{O} \mathrm{III}] \lambda 5007$ to $\mathrm{H} \beta$ is given by

$$
\frac{\mathrm{O}^{+2}}{\mathrm{H}^{+}}=1.8 \times 10^{-4} T_{\mathrm{H} \beta}^{-0.864} T_{5007}^{0.373} \exp \left(\frac{2.888 \times 10^{4}}{T_{5007}}\right)\left[1+1.4 \times 10^{-4} n_{e}\left(\mathrm{O}^{+2}\right) T_{5007}^{-0.5}\right] \frac{I(5007)}{I(\mathrm{H} \beta)},
$$

where $T_{\mathrm{H} \beta}$ and $T_{5007}$ are the temperatures in $\mathrm{H} \beta$ and [O $\left.\mathrm{III}\right] \lambda 5007$, respectively. In the presence of temperature fluctuations, these two temperatures can be calculated from the two equations

$$
T_{5007}^{-0.5} \exp \left(\frac{2.888 \times 10^{4}}{T_{5007}}\right)=T_{0}^{-0.5}\left(\mathrm{O}^{+2}\right) \exp \left[\frac{2.888 \times 10^{4}}{T_{0}\left(\mathrm{O}^{+2}\right)}\right]\left(1+\left\{\left[\frac{2.888 \times 10^{4}}{T_{0}\left(\mathrm{O}^{+2}\right)}\right]^{2}-\frac{8.664 \times 10^{4}}{T_{0}\left(\mathrm{O}^{+2}\right)}+\frac{3}{4}\right\} \frac{t^{2}\left(\mathrm{O}^{+2}\right)}{2}\right)
$$

and

$$
T_{\mathrm{H} \beta}=T_{0}\left(\mathrm{H}^{+}\right)\left[1-0.932 t^{2}\left(\mathrm{H}^{+}\right)\right] .
$$

Equations (A5), (A6), and (A7) give the correct method to determine $\left(\mathrm{O}^{+2} / \mathrm{H}^{+}\right)$when temperature fluctuations are present. The standard method, which assumes $t^{2}=0$, is to substitute $T\left(\mathrm{O}\right.$ III) derived from observations for both $T_{\mathrm{H} \beta}$ and $T_{5007}$ in equation (A5).

Alexander, J., \& Balick, B. 1997, AJ, 114, 713

Aller, L. H. 1990, PASP, 102, 1097

Aller, L. H., \& Czyzak, S. J. 1983, ApJS, 51, 211

Chu, Y.-H., Manchado, A., Jacoby, G. H., \& Kwitter, K. B. 1991, ApJ, 376, 150

Corradi, R. L. M., Perinotto, M., Schwarz, H. E., \& Claeskens, J.-F. 1997, A\&A, 322, 975

Ferland, G. J. 1996, HAZY, Univ. Kentucky Dept. Phys. and Astron. Internal Rep.

Ferland, G. J., Korista, K. T., Verner, D. A., Ferguson, J. W., Kingdon,

J. B., \& Verner, E. M. 1998, PASP, 110, 761

Gruenwald, R., \& Viegas, S. M. 1995, A\&A, 303, 535

Guerrero, M. A., \& Manchado, A. 1996, ApJ, 472, 711

Guerrero, M. A., Stanghellini, L., \& Manchado, A. 1995, ApJ, 444, L49

Iben, I., Kaler, J. B., Truran, J. W., \& Renzini, A. 1983, ApJ, 264, 605

Khromov, G. S. 1989, Space Sci. Rev., 51, 339

Kingdon, J. B., \& Ferland, G. J. 1995, ApJ, 450, 691 (Paper I)

\section{REFERENCES}

Liu, X.-W. 1998, MNRAS, 295, 699

Liu, X.-W., Storey, P. J., Barlow, M. J., \& Clegg, R. E. S. 1995, MNRAS, 272,369

Maciejewski, W., Mathis, J. S., \& Edgar, R. J. 1996, ApJ, 462, 347

Manchado, A., \& Pottasch, S. R. 1989, A\&A, 222, 219

Manchado, A., Pottasch, S. R., \& Mampaso, A. 1988, A\&A, 191, 128

Olive, K. A., Steigman, G., \& Skillman, E. D. 1997, ApJ, 483, 788

Peimbert, M. 1967, ApJ, 150, 825

- 1995, in The Analysis of Emission Lines, ed. R. E. Williams (Cambridge: Cambridge Univ. Press), 165

Peimbert, M., Storey, P. J., \& Torres-Peimbert, S. 1993, ApJ 414, 626

Pérez, E. 1997, MNRAS, 290, 465

Rubin, R. H., et al. 1998, ApJ, 495, 891

Shields, G. A. 1990, ARA\&A, 28, 525

Torres-Peimbert, S., Peimbert, M., \& Peña, M. 1990, A\&A, 233, 540

Vassiliadis, E., \& Wood, P. R. 1993, ApJ, 413, 641 